\title{
Proenkephalin and prognosis in heart failure with preserved ejection fraction: a GREAT network study
}

\author{
Prathap Kanagala ${ }^{1}$ Iain B. Squire ${ }^{1} \cdot$ Donald J. L. Jones ${ }^{1,2} \cdot$ Thong Huy Cao $^{1} \cdot$ Daniel C. S. Chan ${ }^{1} \cdot$ Gerry McCann $^{1}$. \\ Jatinderpal K. Sandhu ${ }^{1}$. Paulene A. Quinn ${ }^{1}$. John McAdam ${ }^{1}$. Anna-Marie Marsh ${ }^{1}$ • Joan E. Davies ${ }^{1}$. \\ Joachim Struck ${ }^{3} \cdot$ Andreas Bergmann $^{3} \cdot$ Zaid Sabti $^{4} \cdot$ Raphael Twerenbold $^{4} \cdot$ Thomas Herrmann $^{4}$. \\ Nikola Kozhuharov ${ }^{4}$ Christian Mueller ${ }^{4}$ Leong L. $\mathrm{Ng}^{1} \cdot$ On behalf of the GREAT (Global REsearch on Acute \\ Conditions Team) Network
}

Received: 25 August 2018 / Accepted: 29 January 2019 / Published online: 14 February 2019

(c) The Author(s) 2019

\begin{abstract}
Background Proenkephalin (PENK), a stable endogenous opioid biomarker related to renal function, has prognostic utility in acute and chronic heart failure. We investigated the prognostic utility of PENK in heart failure with preserved ejection fraction (HFpEF), and its relationship to renal function, Body Mass Index (BMI), and imaging measures of diastolic dysfunction. Methods In this multicentre study, PENK was measured in $522 \mathrm{HFpEF}$ patients (ejection fraction $>50 \%, 253$ male, mean age $76.13 \pm 10.73$ years) and compared to 47 age and sex-matched controls. The primary endpoint was 2-years composite of all-cause mortality and/or heart failure rehospitalisation (HF). A subset $(n=163)$ received detailed imaging studies.

Results PENK levels were raised in HFpEF (median [interquartile range] 88.9 [62.1-132.0]) compared to normal controls (56.3 [47.9-70.5]). PENK was correlated to urea, eGFR, Body Mass Index and $E / e^{\prime}\left(r_{\mathrm{s}} 0.635,-0.741,-0.275,0.476\right.$, respectively, $p<0.0005)$. During 2 years follow-up 144 patients died and 220 had death/HF endpoints. Multivariable Cox regression models showed PENK independently predicted 2 year death/HF [hazard ratio (for $1 \mathrm{SD}$ increment of log-transformed biomarker) HR 1.45 [95\% CI 1.12-1.88, $p=0.005]$ ], even after adjustment for troponin (HR 1.59 [1.14-2.20, $p=0.006]$ ), and Body Mass Index (HR 1.63 [1.13-2.33, $p=0.009]$ ). PENK showed no interaction with ejection fraction status for prediction of poor outcomes. Net reclassification analyses showed PENK significantly improved classification of death/HF outcomes for multivariable models containing natriuretic peptide, troponin and Body Mass Index ( $p<0.05$ for all).

Conclusions In HFpEF, PENK levels are related to BMI, and measures of diastolic dysfunction and are prognostic for allcause mortality and heart failure rehospitalisation.
\end{abstract}

Keywords Heart failure $\cdot$ Preserved ejection fraction $\cdot$ Renal function $\cdot$ B-type natriuretic peptide $\cdot$ Proenkephalin $\cdot$ Opioids

\begin{tabular}{|c|c|c|}
\hline & \multicolumn{2}{|c|}{ Abbreviations } \\
\hline & BMI & Body mass index \\
\hline & BNP & B-type natriuretic peptide \\
\hline & COPD & Chronic obstructive pulmonary disease \\
\hline \multirow{2}{*}{$\begin{array}{l}\text { Leong L. Ng and Christian Mueller are members of the GREAT } \\
\text { network. }\end{array}$} & $\mathrm{HF}$ & Heart failure \\
\hline & HFpEF & Heart failure with preserved ejection \\
\hline
\end{tabular}

Leong L. Ng

lln1@le.ac.uk

1 Department of Cardiovascular Sciences, Clinical Sciences Wing, NIHR Leicester Biomedical Research Centre, Glenfield Hospital, University of Leicester, Leicester LE3 9QP, UK
2 Department of Cancer Studies, Leicester Royal Infirmary, University of Leicester, Leicester LE1 5WW, UK

3 Sphingotec, GmbH, Hennigsdorf, Germany

4 Department of Cardiology, Cardiovascular Research Institute Basel (CRIB), University Hospital Basel, Basel, Switzerland 


\section{LVEF Left ventricular ejection fraction \\ MRI Magnetic resonance imaging \\ NRI Net reclassification improvement \\ NTproBNP N-terminal pro-B-type natriuretic peptide \\ PENK Amino acids 119-159 of proenkephalin A \\ PVD Peripheral vascular disease}

\section{Introduction}

Heart failure prognosis remains poor, with recent studies suggesting a shift in presentations from heart failure with reduced ejection fraction (HFrEF) to those with preserved ejection fraction (HFpEF) [1]. The survival of patients with HFpEF is slightly better than those with HFrEF [2] but has remained unchanged, with improvements seen only in HFrEF due to advances in therapies. Many biomarkers for prognosis in $\mathrm{HFrEF}$ exist, but there are scarce data in HFpEF [3] apart from natriuretic peptides [4]. There is therefore an unmet clinical need for biomarkers in HFpEF that may have a role in patient monitoring [5].

Recent work has suggested a role for the endogenous opioid system (in particular enkephalins) in determining prognosis in a variety of cardiovascular and emergency presentations, including acute myocardial infarction, acute and chronic heart failure (predominantly $\mathrm{HFrEF}$ ), and stroke [6-9]. PENK levels may detect worsening renal function before a rise in plasma creatinine [9]. In cardiac surgery, PENK also predicts acute kidney injury [10]. The cardio-depressor effect of enkephalins on blood pressure and heart rate may play a role in the poor outcomes of these patients, as reviewed recently [11]. In contrast, enkephalins may also be a counter-regulatory mechanism (e.g. through the delta receptor which is highly expressed in kidney tissue [12], enkephalins may alter renal blood flow, increase diuresis and natriuresis, and could also be cardioprotective [11]). In all these studies, a stable peptide precursor of PENK [13] has been investigated since the enkephalins are unstable in plasma.

In HFpEF, kidney dysfunction is related to mortality [14]. Due to the links of enkephalins with cardiorenal status and existing evidence documenting the relationship of PENK and prognosis of cardiovascular disease, we examined the role of PENK in HFpEF. Previous studies on biomarkers in HFpEF have used a variety of cutoff values for left ventricular ejection fraction (LVEF) varying between 40 and 50\%, but more recent guidelines have defined HFrEF and HFpEF as patients having ejection fractions under $40 \%$ and over $50 \%$, respectively [15]. In the present study, we have used these ejection fraction cutoff values for patient recruitment, and investigated the relationship of the enkephalin system and renal function to prognosis in HFpEF. We have also explored the link of PENK to echocardiographic and cardiac MRI indices of diastolic dysfunction.

\section{Methods}

\section{Study population}

Two cohorts of HFpEF patients were recruited in university hospitals in Leicester, UK and Basel, Switzerland between February 2006 and August 2011, with 73\% participating as in-patients and the rest as out-patients. The patients were acutely decompensated chronic HF patients or acute HF patients. Cardiac decompensation was defined as hospitalisation with HF as the primary reason, requiring treatment with diuretics, intravenous inotropes or nitrates. All patients had clinical or radiographic evidence of $\mathrm{HF}$, age $\geq 18$ years, left ventricular ejection fraction (LVEF) $\geq 50 \%$ on transthoracic echocardiography (TTE), elevated natriuretic peptide levels and/or structural heart disease such as left ventricular hypertrophy or left atrial enlargement, to define HFpEF [15].

For comparison with HF, 47 healthy asymptomatic controls (age and sex-matched) were also recruited. We included hypertensive controls $(n=22)$ as a major proportion of HFpEF patients had hypertension. We also compared the findings in HFpEF to 667 patients with HFrEF who had echocardiography studies showing LVEF $<40 \%$ (part of a cohort reported previously [9]). The exclusion criteria were: known myocardial infarction in the preceding 6 months, suspected or confirmed cardiomyopathy or constrictive pericarditis, non-cardiovascular life expectancy $<6$ months, and being on renal replacement therapy. This study complied with the declaration of Helsinki and ethics approval was granted from respective research ethics committees. All patients provided written informed consent.

\section{Plasma sampling}

Venous blood was withdrawn from recumbent patients and collected in pre-chilled tubes containing EDTA to prepare plasma. Plasma was stored at $-80{ }^{\circ} \mathrm{C}$ until analysis in a single batch.

\section{Echocardiography and magnetic resonance imaging}

Transthoracic echocardiography was performed using standard techniques [16] and left ventricular ejection fraction (LVEF) was calculated using the biplane method of discs formula. Based on doppler mitral inflow, the following measurements were derived: peak of early filling ( $E$ velocity), peak of late atrial filling ( $A$ velocity) and $E / A$ ratio.

In a subset of 163 patients, tissue Doppler measurements were available, and $E / e^{\prime}$ was calculated from the mitral inflow velocity of the $E$ wave, divided by the mitral annular 
velocity. Patients with prosthetic valves or valve calcification were not studied. The medial and lateral $E / e^{\prime}$ values were averaged and used for analysis. In 108 patients, MRI scanning was undertaken and analysed as previously described [17]. The biplane area-length method (excluding the appendage and pulmonary veins) was employed for LA volumetric analysis [18].

\section{PENK assay}

A modified version of the original assay for a stable peptide derived from preproenkephalin A (amino acids 119-159 of proenkephalin $\mathrm{A}$, molecular weight $4586 \mathrm{Da}$ ) was used $[8,13]$. A mouse monoclonal antibody against this peptide was used as the capture antibody, and another mouse monoclonal antibody labelled with methyl-acridinium ester was used as the detector, with bound chemiluminescence measured. In plasma from normal controls, PENK values were mean \pm SEM $46.6 \pm 14.1 \mathrm{pmol} / \mathrm{L}$, with a median [range] of 45 [9-518] pmol/L.

\section{Other biomarker assays}

In Leicester, the Centaur cTnI Ultra immunoassay (Siemens Healthcare Diagnostics) was used to measure troponin I (with 99th percentile of $0.04 \mu \mathrm{g} / \mathrm{L}$ ). In Basel, plasma hsTnT (Roche Elecsys Assay; Roche Diagnostics GmbH, Mannheim, Germany) was measured (with 99th percentile of $0.014 \mu \mathrm{g} / \mathrm{L})$.

For the natriuretic peptides, plasma NTproBNP was quantified using a sandwich immunoassay in Leicester, as described previously [19]. The Roche Elecsys NTproBNP assay was used in Basel. To normalise data from different assays, we log-transformed the natriuretic peptide and troponin values and calculated the $\mathrm{Z}$ transform for each site (dividing by $1 \mathrm{SD}$ ) before combining these values for analysis.

\section{Outcomes}

All patients had a minimum follow-up of at least 2 years. The primary endpoint was all-cause mortality or heart failure (HF) rehospitalisation within 2 years. HF rehospitalisation was defined as a hospital readmission for which HF was the primary reason, requiring treatment with diuretics, intravenous inotropes or nitrates. Endpoints were obtained from hospital records and electronic databases. Secondary endpoints included all-cause mortality at 2 years. In cases with multiple events, the time to first event was used as the censored outcome.

\section{Statistical analysis}

Statistical analyses were performed on SPSS Version 24 (SPSS Inc, Chicago, IL) and Stata 14 (TX, USA). Assuming an event rate of $40 \%$ at 2 years, a sample size of 500 patients would be powered $(93.5 \%$ at $p<0.05)$ to detect a hazard ratio of the biomarker of 1.4, using the command stpower cox in Stata 14. Normality of distribution was assessed by visual inspection of plotted histograms and $Q Q$ plots and by the Kolmogorov-Smirnov test. Normally and non-Gaussian distributed variables were reported as mean (SD) or median (interquartile range), respectively. All biomarker levels were $\log _{10}$ transformed and normalised to 1 SD increment.

Normalised data were analysed using ANOVA and general linear models, and $p$ values were Bonferroni-corrected for multiple comparisons. Non-Gaussian data and categorical variables were analysed using non-parametric tests [Mann-Whitney $U$ test, Kruskal-Wallis test and Spearman $\left(r_{\mathrm{s}}\right)$ correlations] and $\chi^{2}$ tests, respectively. Variables and factors that independently predict PENK levels were investigated using general linear models bootstrapped 1000 times. All models were based on cases with complete data.

Cox survival analysis was used to define a base model, including variables associated (at $p<0.10$ ) with the primary composite outcome of death and/or HF and the secondary outcome of death. Hazard ratios refer to unit changes in age (years), eGFR $\left(\mathrm{mL} / \mathrm{min} 1.73 \mathrm{~m}^{2}\right), \mathrm{BP}(\mathrm{mmHg})$, and heart rate (beats/min). Z-transformed natriuretic peptide or PENK levels were added to the base model to assess their prognostic performance, so that hazard ratios are normalised to $1 \mathrm{SD}$ increment of the log-transformed biomarkers.

Kaplan-Meier survival analysis was used to visualise cumulative survival and significance between tertiles tested using log rank tests. The probabilities of outcomes derived from logistic regression analysis were used in reclassification analysis using category-free net reclassification improvement (NRI) as described by Pencina et al. [20] to assess biomarker performance in up- or down-classifying risk in those with and without endpoints at 2 years.

\section{Results}

\section{Patient characteristics}

Patient characteristics from the two centres are reported in Supplementary Table 1. Basel patients were on average older and had lower Body Mass Index (BMI), with higher prevalences of ischemic heart disease, renal failure, hypertension, chronic obstructive pulmonary disease (COPD) and peripheral vascular disease (PVD) and less diabetes. Plasma urea, creatinine and PENK were higher in Basel patients, although 
eGFR was similar in both centres. Leicester patients received less $\beta$-blockers and more aldosterone receptor antagonists.

Patients with HFpEF were compared to normal controls without HF. Differences in age, sex distribution, and Body Mass Index are reported in Supplementary Table 2. HFpEF patients also had elevated $E / e^{\prime}$ and PENK levels compared to normal controls $(p<0.0005)$. Patients with HFpEF were older, had higher BMI and with a female preponderance compared to HFrEF patients. HFrEF patients had a higher proportion of ischaemic heart disease aetiology and lower hypertension prevalence, and had poorer renal function.

HFpEF patient characteristics stratified according to PENK tertiles are reported in Table 1. Patients in the highest PENK tertile were older and had lower BMI, and had greater prevalence of ischemic heart disease, renal failure, hypertension and lower prevalence of AF. Renal dysfunction and higher NTproBNP levels were evident in the highest PENK tertile. There were no therapy differences between PENK tertiles, apart from aspirin, which may reflect the differing prevalence of ischemic heart disease. Death and the composite endpoint of death /HF were also more prevalent in the highest PENK tertile.

\section{Correlation analysis}

PENK was correlated to age $\left(r_{\mathrm{s}} 0.363, p<0.0005\right)$, Body Mass Index (BMI) $\left(r_{\mathrm{s}}-0.275, p<0.0005, n=411\right)$, eGFR $(-0.741, p<0.0005)$, plasma creatinine $(0.667, p<0.0005)$, plasma urea $(0.635, p<0.0005), Z$ score of $\log$ natriuretic peptides $(0.437, p<0.0005)$, heart rate $(-0.094, p=0.032)$ and systolic BP $(-0.145, p=0.001)$. The relationship of PENK with eGFR or BMI in HFpEF is illustrated in Supplementary Fig. 1.

Multivariable general linear modelling was used to determine variables that showed independent association with PENK. Supplementary Table 3 shows the independent significant predictors were plasma urea, eGFR, natriuretic peptide levels, age, AF, previous history of IHD, and renal failure. In the subset $(n=411)$ for which BMI was available, BMI was retained as an independent predictor with the above variables, whilst age and AF were excluded. The predictors accounted for $64-69 \%$ of the variance in PENK levels.

\section{Echocardiographic and magnetic resonance imaging parameters: relationship with PENK levels}

In a subset of patients in whom tissue Doppler measurements were available, PENK was correlated to $E / e^{\prime}(0.453$, $p<0.0005, n=163$ : Fig. 1$)$. The mean (SD) for $E / e^{\prime}$ according to increasing PENK tertiles was 10.67 (3.42), 14.14 (5.51), and 16.57 (6.58), respectively, [ANOVA $p<0.0005$, with the top tertile statistically different from the 1st tertiles (Bonferroni-corrected $p<0.0005)$ and 2nd $(p=0.046)]$.

Cardiac magnetic resonance imaging (MRI) left atrial volume indices in systole and diastole (LAEDVI, LAESVI) were available in a subset of $108 \mathrm{HFpEF}$ patients. Figure 2 shows box plots of these volumes according to PENK tertiles. LAEDVI and LAESVI differed between PENK tertiles (ANOVA $p=0.024$ and 0.01 ). The second tertile of LAEDVI was higher than the first $(p=0.025)$. Both second and third tertiles of LAESVI were higher than the first $(p=0.016$ and 0.042 , respectively). The MRI derived left ventricular end diastolic and end systolic mass indices (LVEDMI and LVESMI) were similar between the PENK tertiles, as were the echocardiography-measured LV mass indices and LVEDM/LVEDV ratio ( $p$ non-significant).

\section{Survival analysis}

During follow-up for 2 years, there were 144 deaths and 220 death/HF endpoints. As PENK tertile increased, so did the incidence of death alone or of the composite end-point of death or heart failure hospitalisation. Cox proportional hazard survival modelling was used to investigate factors associated with the outcome of death/HF at 2 years, and hazard ratios for PENK (for $1 \mathrm{SD}$ increment of the log-transformed biomarker) are reported in Fig. 3.

The base model contained the variables age, gender, NYHA class IV, past history of heart failure, ischemic heart disease, hypertension, diabetes, atrial fibrillation, systolic $\mathrm{BP}$, heart rate, plasma urea, creatinine, sodium, haemoglobin, and natriuretic peptide. This contained variables that have been related to outcome, or had univariable association with the outcome $(p<0.05)$. Univariable hazard ratios for the base model variables are reported in Supplementary Table 4. The HR for PENK (univariable HR A in Fig. 3), remained significant when adjusted for the base model (HR B in Fig. 3). The HR for PENK was significant on further adjustment with the base model and troponin (HR C in Fig. 3), and on addition of BMI to the base model with troponin (HR D in Fig. 3).

Cox survival modelling of HFrEF patients showed PENK remained significant on adjustment with the base model variables, although not following further adjustment with addition of troponin and BMI to the models. On interaction analysis in the whole population, PENK hazard ratios remained significant following adjustment using the base model, troponin, and BMI. Interaction terms of PENK with $\mathrm{HFpEF} / \mathrm{HFrEF}$ status were non-significant suggesting similar performance of the biomarker irrespective of ejection fraction.

The lower panel of Fig. 3 illustrates the hazard ratios of PENK for death at 2 years (unadjusted, A) and following 
Table 1 Clinical characteristics of HFpEF patients

\begin{tabular}{|c|c|c|c|c|c|}
\hline & All & $\begin{array}{l}1 \\
<68.2 \mathrm{pmol} / \mathrm{L}\end{array}$ & $\begin{array}{l}2 \\
68.2-111.6 \mathrm{pmol} / \mathrm{L}\end{array}$ & $\begin{array}{l}3 \\
>111.6 \mathrm{pmol} / \mathrm{L}\end{array}$ & $p$ value \\
\hline Number & $n=522$ & $n=174$ & $n=174$ & $n=174$ & \\
\hline \multicolumn{6}{|l|}{ Demographics } \\
\hline Age (years) & $76.13(10.73)$ & $71.29(11.50)$ & $76.91(9.79)$ & $80.19(8.82)$ & $<0.0005$ \\
\hline Male $(\%)$ & $253(48.5)$ & $91(52.2)$ & $83(47.7)$ & $79(45.4)$ & 0.424 \\
\hline Body Mass Index $\left(\mathrm{kg} / \mathrm{m}^{2}\right)$ & $30.10(6.89)$ & $31.91(7.20)$ & $30.02(6.15)$ & $28.11(6.70)$ & $<0.0005$ \\
\hline \multicolumn{6}{|l|}{ Previous history } \\
\hline Ischemic heart disease & $171(32.7)$ & $41(23.6)$ & $63(36.2)$ & $67(38.5)$ & 0.006 \\
\hline $\begin{array}{l}\text { Renal failure } \\
\quad(\mathrm{eGFR}<90 \mathrm{~mL} / \\
\left.\mathrm{min} / 1.73 \mathrm{~m}^{2}\right)\end{array}$ & $155(29.7)$ & $14(8.0)$ & $41(23.5)$ & $100(57.5)$ & $<0.0005$ \\
\hline Heart failure & $243(46.7)$ & 74 (42.5) & 83 (47.7) & $86(50.0)$ & 0.361 \\
\hline Hypertension & $425(81.4)$ & $130(74.7)$ & $147(84.4)$ & $148(85.1)$ & 0.021 \\
\hline Diabetes mellitus & $189(36.2)$ & $67(38.5)$ & $59(33.9)$ & $63(36.2)$ & 0.672 \\
\hline $\mathrm{AF}$ & $222(42.6)$ & $81(46.6)$ & $88(50.6)$ & $53(30.6)$ & $<0.0005$ \\
\hline Stroke & $82(15.7)$ & $22(12.6)$ & $25(14.4)$ & $35(20.2)$ & 0.126 \\
\hline COPD & $87(16.7)$ & $35(20.1)$ & $23(13.2)$ & $29(16.8)$ & 0.226 \\
\hline PVD & $61(11.7)$ & $15(8.6)$ & $18(10.3)$ & $28(16.2)$ & 0.072 \\
\hline \multicolumn{6}{|l|}{ Initial observations } \\
\hline Systolic BP (mmHg) & $142.68(26.70)$ & $145.58(25.27)$ & $144.53(26.16)$ & $137.92(28.11)$ & 0.015 \\
\hline Heart rate (beats/min) & $83.44(25.03)$ & $85.05(25.53)$ & $84.53(24.78)$ & $80.73(24.70)$ & 0.218 \\
\hline Ejection fraction (\%) & $58.46(6.29)$ & $57.93(6.18)$ & $58.03(5.85)$ & $59.43(6.76)$ & 0.049 \\
\hline NYHA class $1(\%)$ & $46(8.9)$ & $27(15.5)$ & $17(9.9)$ & $2(1.1)$ & $<0.0005$ \\
\hline NYHA class $2(\%)$ & $90(17.4)$ & $34(19.5)$ & $33(19.2)$ & $23(13.5)$ & 0.391 \\
\hline NYHA class $3(\%)$ & $235(45.5)$ & $73(41.9)$ & $77(44.3)$ & $90(51.7)$ & 0.161 \\
\hline NYHA class $4(\%)$ & $146(28.2)$ & $40(22.9)$ & $47(27.3)$ & $59(34.5)$ & 0.056 \\
\hline \multicolumn{6}{|l|}{ Plasma biomarkers } \\
\hline Urea (mmol/L) & $9.79(5.51)$ & $6.70(2.13)$ & $8.49(3.24)$ & $14.29(6.77)$ & $<0.0005$ \\
\hline Creatinine $(\mu \mathrm{mol} / \mathrm{L})$ & $113.89(56.81)$ & $82.59(21.52)$ & $101.95(29.59)$ & $156.89(73.28)$ & $<0.0005$ \\
\hline eGFR $\left(\mathrm{mL} / \mathrm{min} / 1.73 \mathrm{~m}^{2}\right)$ & $59.98(24.95)$ & $78.33(22.69)$ & $61.33(18.28)$ & $40.39(17.32)$ & $<0.0005$ \\
\hline Haemoglobin $(\mathrm{g} / \mathrm{L})$ & $123.6(20.8)$ & $133.3(19.6)$ & $123.5(18.8)$ & $114.0(19.5)$ & $<0.0005$ \\
\hline Troponin I $(\mu \mathrm{g} / \mathrm{L})$ & $0.17(0.58)$ & $0.18(0.59)$ & $0.19(0.62)$ & $0.14(0.52)$ & 0.082 \\
\hline Sodium (mmol/L) & $138.16(5.03)$ & $138.54(4.68)$ & $137.97(5.53)$ & $137.97(4.84)$ & 0.481 \\
\hline $\begin{array}{l}\text { NTproBNP }(\mathrm{pmol} / \mathrm{L}) \\
\text { Leicester }\end{array}$ & 2312.6 [1128.7-4143.7] & 650.6 [77.7-1817.3] & 1048.6 [479.9-2205.9] & 1569.6 [729.7-2876.3] & 0.008 \\
\hline $\begin{array}{l}\text { NTproBNP(pg/mL) } \\
\text { Basel }\end{array}$ & $4897.5[2653.5-10,572.5]$ & 1673.5 [644-3431.5] & 3281.5 [1484.3-4837.5] & $6365.5[3121.5-10,827.3]$ & $<0.0005$ \\
\hline \multicolumn{6}{|l|}{ Treatment } \\
\hline Aspirin & $194(37.5)$ & 48 (27.6) & $75(43.1)$ & $71(41.8)$ & 0.004 \\
\hline$\beta$-Blocker & $326(62.9)$ & $103(59.2)$ & $113(64.9)$ & $110(64.7)$ & 0.456 \\
\hline ACE inhibitor or $\mathrm{ARB}$ & $390(74.7)$ & $134(77.0)$ & $136(78.2)$ & $120(69.0)$ & 0.099 \\
\hline Aldosterone antagonists & $64(12.2)$ & $23(13.2)$ & $27(15.5)$ & $14(8.0)$ & 0.094 \\
\hline Statin & $183(48.3)$ & $45(42.1)$ & 65 (49.6) & $73(51.8)$ & 0.295 \\
\hline Digoxin & $61(11.8)$ & $20(11.5)$ & $24(13.8)$ & $17(10.0)$ & 0.546 \\
\hline \multicolumn{6}{|l|}{ End points ( 2 years) } \\
\hline Death & $144(27.7)$ & $25(14.4)$ & $44(25.4)$ & $75(43.1)$ & $<0.0005$ \\
\hline Death and/or heart failure & $220(42.1)$ & $45(25.9)$ & $76(43.7)$ & $99(56.9)$ & $<0.0005$ \\
\hline
\end{tabular}

Characteristics of the $522 \mathrm{HFpEF}$ patients in the Leicester and Basel cohorts, according to PENK tertiles. NTproBNP is reported for the cohorts, respectively. Body Mass Index measurements were available in 411 cases. Numerical data are presented as $n(\%)$ and mean (SD) or median (Interquartile range) are reported. $p$ values are quoted for the ANOVA/Kruskal Wallis or Chi squared tests for continuous or categorical variables, respectively

$A R B$ angiotensin 2 receptor blocker 


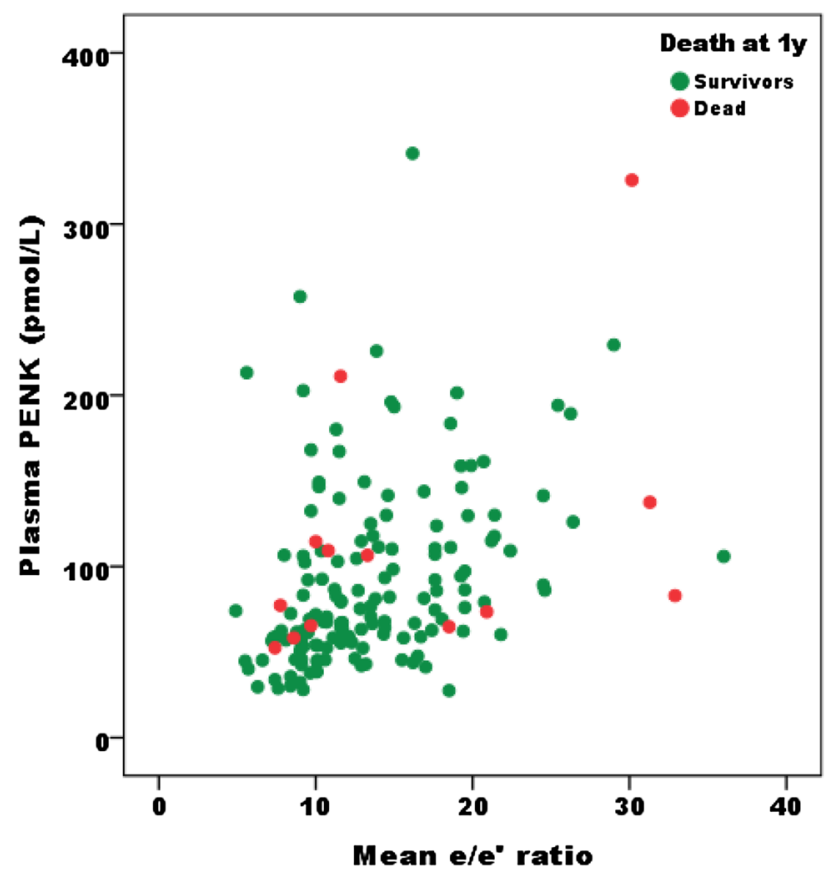

Fig. 1 PENK relationship to the echocardiographic diastolic index $E / e^{\prime}$. Correlation of PENK with echocardiographic index of diastolic dysfunction $E / e^{\prime}$. The Spearman correlation coefficient was 0.453 $(p<0.0005)$

adjustment using the base model (B), with further additions of troponin (C) or BMI (D). PENK hazard ratios were significant following adjustment using the base model but not with further addition of troponin or BMI. Interaction analysis suggested PENK had similar performance irrespective of ejection fraction.

Kaplan-Meier analysis (Fig. 4) of the HFpEF cohort showed patients in the highest tertile had worse outcomes than those in tertile $1(p<0.0005)$ or $2(p=0.006)$ for the endpoint of death/HF. For all-cause mortality, patients in the highest tertile had worse survival than those in tertile 1 $(p<0.0005)$ or $2(p<0.0005)$.

\section{Reclassification analyses and $C$ statistics}

Logistic regression model derived risk scores for death/HF at 2 years using base model variables with further addition of troponin and BMI, were used with addition of PENK to calculate the continuous net reclassification improvement index NRI ( $>0$ ) (Table 2). PENK showed significant net reclassification improvement on the base model, and on addition of troponin and BMI.

For the outcome of death at 2 years, PENK showed significant net reclassification improvement on the base model, but not when troponin or BMI were added to the base model. The increments in $\mathrm{C}$ statistic on addition of PENK to the base model, or models with troponin and BMI were not significant. Areas under the receiver operating characteristic curves for PENK, natriuretic peptides, troponin and the combination of all three for the outcomes of death/HF or death at 2 years are illustrated in Supplementary Fig. 2.

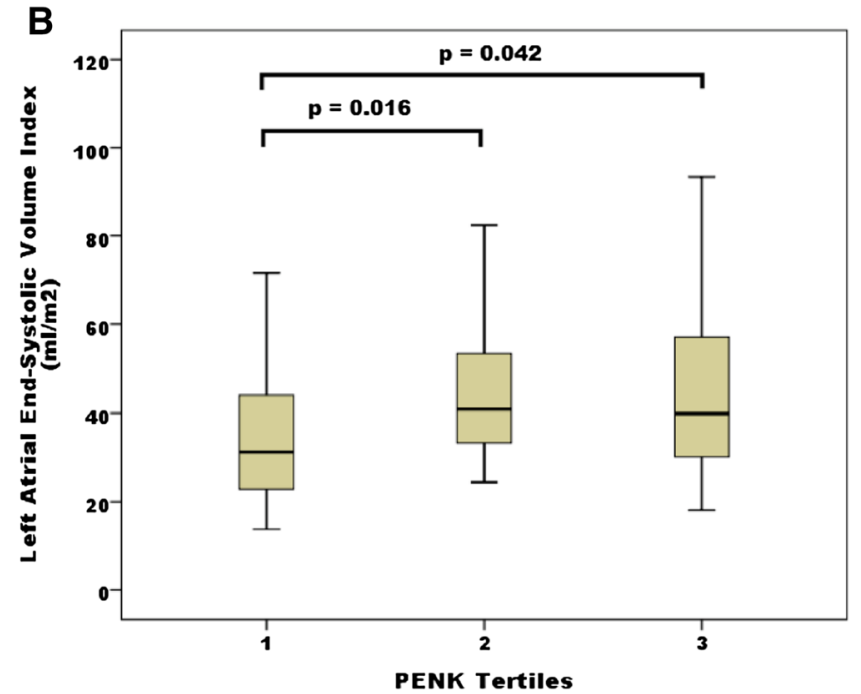

seen between the second and first tertile of LAEDVI $(p=0.025)$ and between second and third tertiles of LAESVI and the first tertile $(p=0.016$ and 0.042 , respectively)
Fig. 2 MRI-derived ventricular volumes according to PENK tertiles. Box and whisker plots of a LAEDVI and b LAESVI according to PENK tertiles. LAEDVI and LAESVI differed between PENK tertiles (ANOVA $p=0.024$ and 0.01). Significant differences were

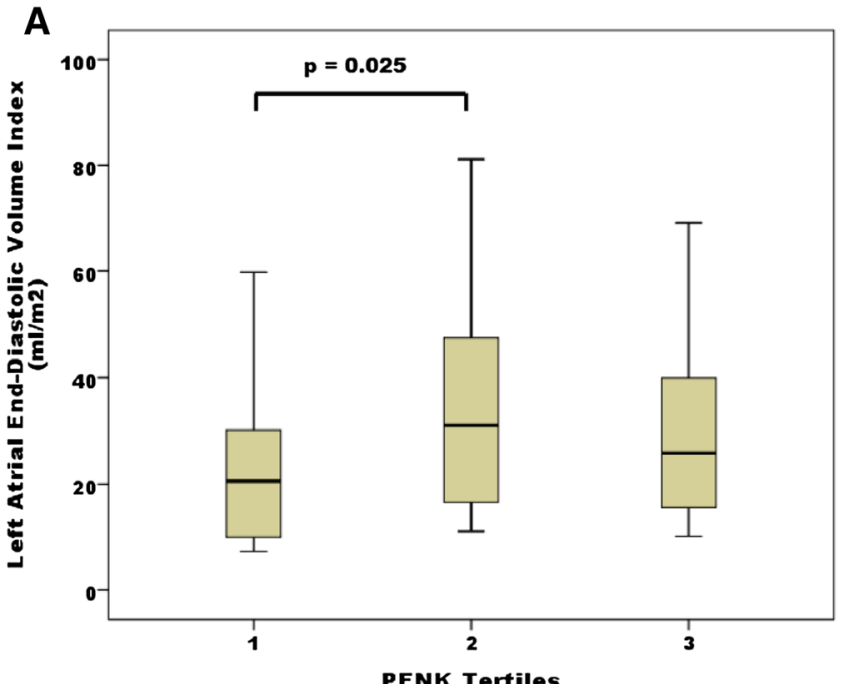




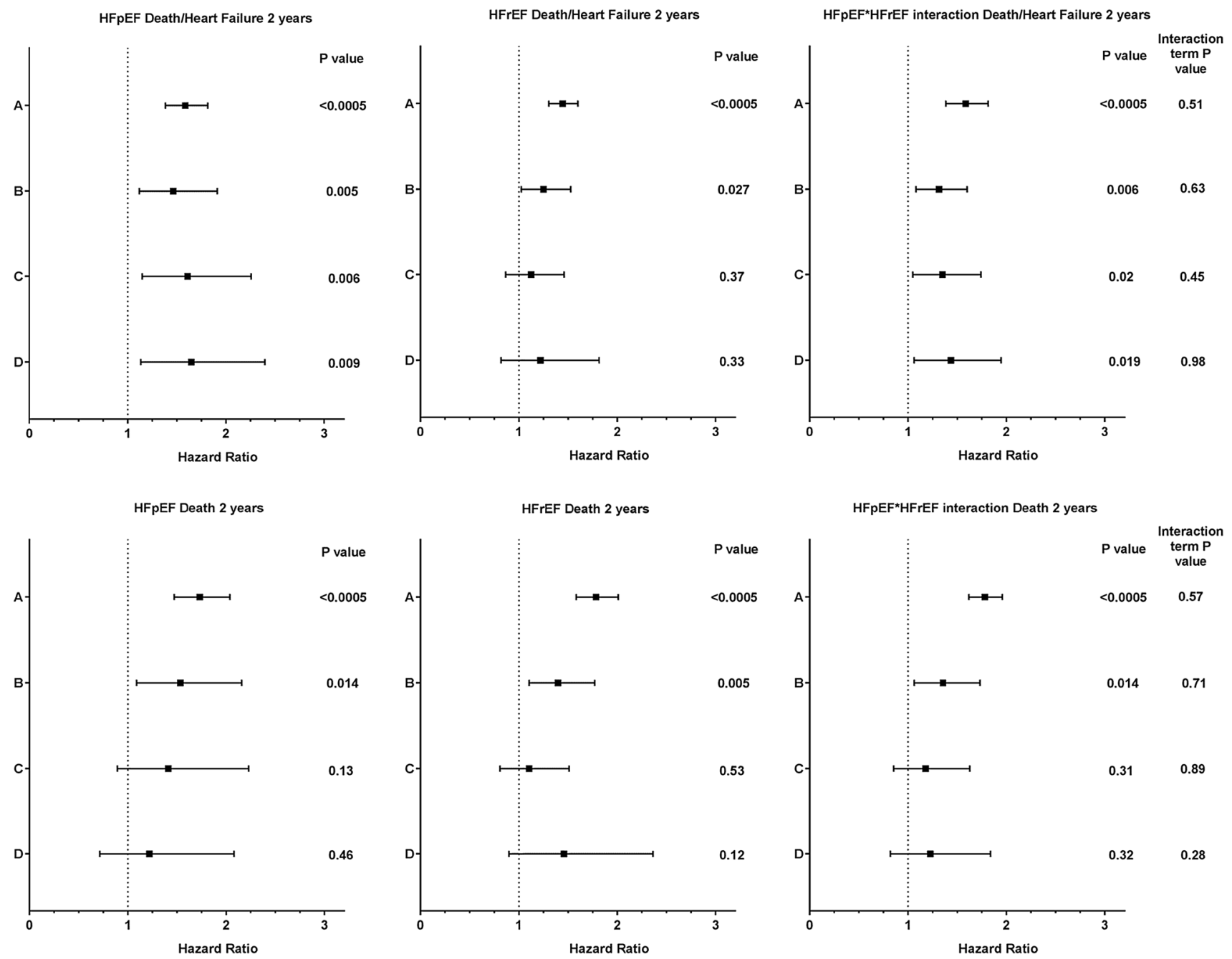

Fig. 3 Hazard ratios for PENK in Cox survival analysis for Death/HF or Death at 2 years in HFpEF and HFrEF. Forest plots showing hazard ratios and confidence intervals for PENK (as a univariable in A) following adjustment for a multivariable base model $(\mathrm{B}$, containing the variables age, gender, NYHA class IV, past history of heart fail-

\section{Discussion}

Although many biomarkers have been described for diagnosis or prognosis in HFrEF, few biomarkers in HFpEF perform beyond base models of clinical variables [3]. Natriuretic peptides [4] have been shown to independently predict outcomes in HFpEF. However, many previous reports were based on clinical trials, and may not have used the contemporary definition of cutoff values of ejection fraction for HFpEF (ejection fraction $\geq 50 \%$ ) [15]. There is a clinical need for such biomarkers in HFpEF as they may facilitate clinical care, as well as the search for therapies that may influence outcomes.

In this study of HFpEF patients, as defined by contemporary cutoff values in ejection fraction, we have confirmed ure, ischemic heart disease, hypertension, diabetes, atrial fibrillation, systolic BP, heart rate, plasma urea, creatinine, sodium, haemoglobin, natriuretic peptide), base model with troponin (C), and base model with troponin and BMI (D). Hazard ratios for interaction of PENK with ejection fraction status are shown on the right of the figure

that PENK is a strong correlate of renal function, and provides prognosis for the composite outcome of death and/or HF hospitalisation. In these multivariable models, PENK emerged as a significant marker for death/HF, even following adjustment for clinical variables that have previously been reported as prognostic markers, such as AF [21] and anaemia [22]. PENK remained an independent marker for death/HF even following adjustment for troponin and Body Mass Index. The performance of PENK as a prognostic marker for death/HF was independent of ejection fraction, as there was no significant interaction with ejection fraction status (reduced or preserved). We also used reclassification analysis [20], which confirmed the prognostic performance of PENK for the composite death/HF endpoint. For the endpoint of death alone, PENK remained a significant 
A Death/HF endpoint

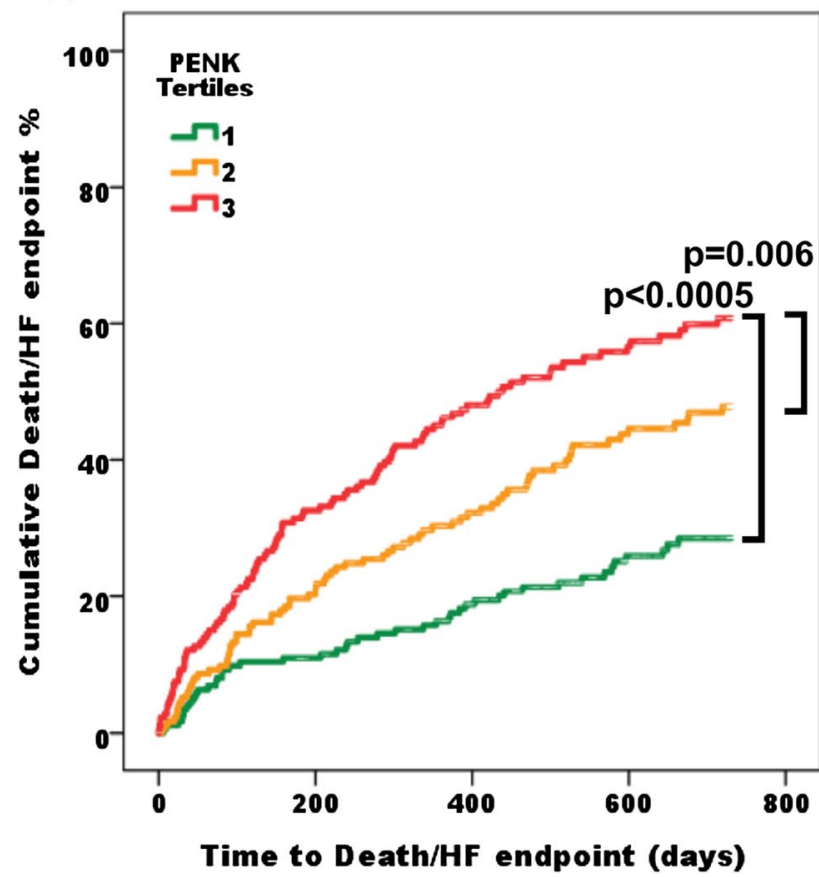

Fig. 4 Kaplan-Meier survival analysis for death/HF hospitalisation and all-cause mortality. Kaplan-Meier plots of a the composite endpoint of death and/or HF hospitalization and $\mathbf{b}$ all-cause mortal-

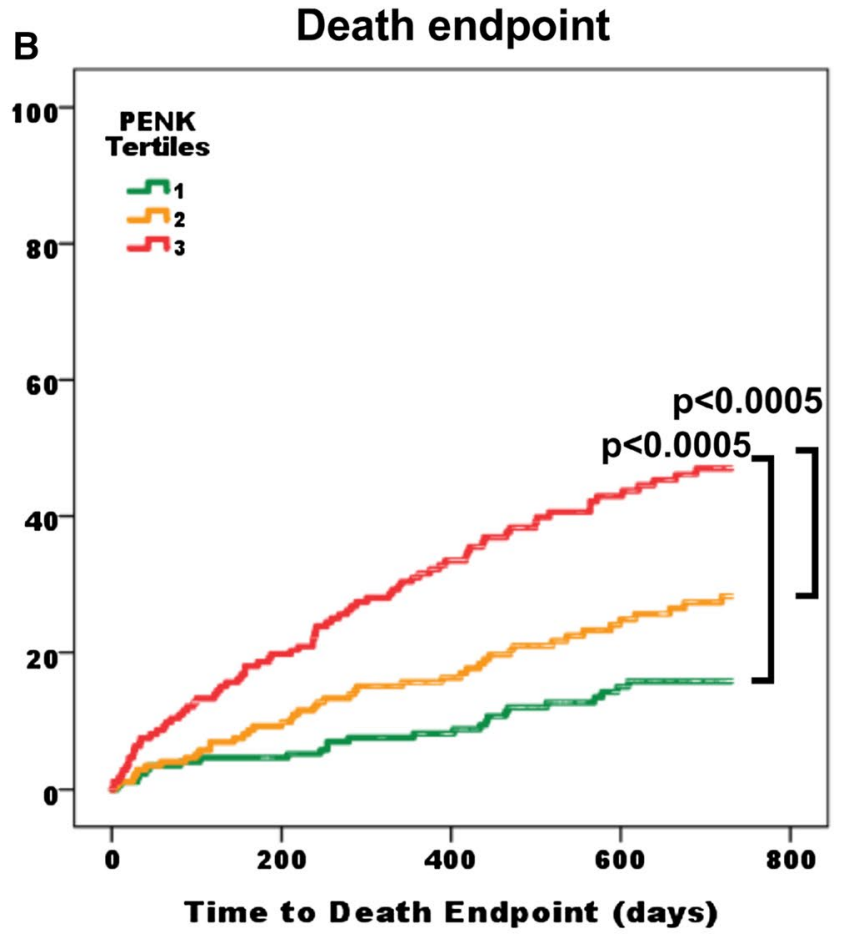

ity, according to PENK tertiles. Log rank tests showed differences between tertiles 1 and 3 ( $p<0.0005$ for both endpoints), and between tertiles 2 and 3 ( $p=0.006$ for death/HF and $p<0.0005$ for death)
Table $2 C$ statistics and reclassification analysis for death/HF or death at 2 years using biomarkers

\begin{tabular}{|c|c|c|c|c|c|}
\hline \multirow[t]{2}{*}{ Model } & \multirow{2}{*}{$\begin{array}{l}C \text { statistic }(95 \% \\
\text { confidence } \\
\text { interval) }\end{array}$} & \multirow{2}{*}{$\begin{array}{l}\text { Model + PENK } C \text { statistic } \\
\text { (95\% confidence interval) }\end{array}$} & \multirow[t]{2}{*}{$P$ value } & \multicolumn{2}{|l|}{ Reclassification analysis } \\
\hline & & & & NRI (95\% confidence interval) & $P$ value \\
\hline \multicolumn{6}{|c|}{ Death/HF 2 years } \\
\hline A & $0.69(0.63-0.75)$ & & & & \\
\hline $\mathrm{B}$ & $0.69(0.63-0.75)$ & $0.70(0.64-0.77)$ & NS & $18.9(0.10-37.8)$ & 0.049 \\
\hline $\mathrm{C}$ & $0.70(0.64-0.76)$ & $0.72(0.66-0.78)$ & NS & $24.4(2.2-46.5)$ & 0.031 \\
\hline $\mathrm{D}$ & $0.70(0.64-0.76)$ & $0.72(0.66-0.78)$ & NS & $26.6(2.7-50.5)$ & 0.029 \\
\hline \multicolumn{6}{|c|}{ Death 2 years } \\
\hline A & $0.66(0.61-0.75)$ & & & & \\
\hline B & $0.79(0.73-0.85)$ & $0.79(0.72-0.85)$ & NS & $22.3(0.50-44.0)$ & 0.045 \\
\hline $\mathrm{C}$ & $0.81(0.75-0.87)$ & $0.81(0.75-0.87)$ & NS & $11.5(-14.6$ to 37.5$)$ & 0.38 \\
\hline D & $0.82(0.76-0.88)$ & $0.82(0.76-0.88)$ & NS & $-11.1(-40.3$ to 18.0$)$ & 0.45 \\
\hline
\end{tabular}

$\mathrm{C}$ statistics and reclassification analysis, for the outcomes of death/HF or death at 2 years, using continuous reclassification showing the net reclassification improvement of adding PENK to the base model, and models with troponin and BMI

A, univariable PENK $C$ statistic

$\mathrm{B}$, base model (containing variables age, gender, NYHA class IV, past history of heart failure, ischemic heart disease, hypertension, diabetes, atrial fibrillation, systolic BP, heart rate, plasma urea, creatinine, sodium, haemoglobin, and natriuretic peptide)

C, base model with troponin

$\mathrm{D}$, base model with troponin and BMI 
prognostic marker following addition to the base model, but not when troponin was added to the model. However, PENK did not improve the $\mathrm{C}$ statistic significantly for poor outcomes when added to any of the models. It has been reported that reclassification analysis is more sensitive and less conservative when assessing utility of biomarkers in models [23].

We have previously reported PENK as a predictor of poor outcomes in acute heart failure [9] (predominantly HFrEF and HFmEF as defined in current guidelines [15]), and in acute myocardial infarction [8], and the association with worsening renal function [8]. The current findings complement these previous reports. Enkephalins have a cardiodepressor effect with a negative inotropic effect, lowered BP and reduced tissue perfusion $[11,24]$. Reduced renal perfusion would be a potential mechanism by which PENK might directly influence eGFR. The current findings reinforce these previous reports of the strong association of PENK with renal function and poor outcomes, and may suggest the PENK system as a suitable target for therapeutic intervention, as well as a marker of prognosis, in HFpEF.

In a subgroup of these HFpEF patients, we have demonstrated a correlation of PENK with the tissue Doppler echocardiography parameter $\mathrm{E} / \mathrm{e}^{\prime}$, a measure of the left ventricular filling pressure that reflects diastolic dysfunction. Further evidence in support of this was the larger left atrial volume indices seen in the second and third tertiles of PENK.

Body Mass Index was available in a subset of the HFpEF patients, and a negative correlation was observed with PENK levels, resembling the findings with natriuretic peptides. This relationship was independent as general linear modelling retained BMI in addition to renal function measures, natriuretic peptide levels and previous history of renal failure and ischemic heart disease. A previous study has also noted a negative relationship between PENK and weight and body surface area [6]. Enkephalins are widely distributed in many tissues [12] and have a lipolytic effect on adipose tissue [25]. A recent finding may be of relevance to this negative association of enkephalins with body weight, as group 2 innate immune cells (ILC2) may have a "beiging" effect on white adipose, converting it to higher energy expending brown fat with met-enkephalin as the likely candidate factor [26].

\section{Limitations}

Our findings are based on two European centres recruiting heart failure patients and should be validated in additional, non-European populations. While there were clinically relevant differences in patient characteristics between the two centres, our findings are based on models corrected for these differences. Different natriuretic peptide assays were used, and we tried to mitigate this using $Z$ transforms of the logged natriuretic peptide levels. Body Mass Index was not available on all patients and tissue Doppler echocardiography and MRI studies were only performed on a subgroup of patients. The patients who underwent cardiac MRI were younger and had better renal function than the rest of the cohort, since renal impairment precluded the use of MRI contrast.

\section{Conclusions}

In HFpEF, PENK provides prognostic information on the composite outcome of all-cause mortality and/or heart failure rehospitalisation, reflecting echocardiographic measures of diastolic dysfunction. High PENK levels are associated with increased ventricular and atrial volume indices, lowered Body Mass Index and renal impairment.

Acknowledgements This work was supported by the John and Lucille Van Geest Foundation, British Heart Foundation (FS/15/10/31223) and the National Institute for Health Research Leicester Biomedical Research Centre. Sphingotec $\mathrm{GmbH}$ is a midsized company based in Hennigsdorf, Germany; it commercializes immunoassays, and has developed the Proenkephalin assay, for which it owns patent rights.

\section{Compliance with ethical standards}

Conflict of interest Dr. Bergmann holds ownership in Sphingotec $\mathrm{GmbH}$ which manufactures the PENK assay, and is a member of the board of directors of Sphingotec GmbH. Dr. Struck is an employee of Sphingotec GmbH which manufactures the PENK assay. Dr. Mueller has received research grants from the Swiss National Science Foundation, the Swiss Heart Foundation, the European Union, the Cardiovascular Research Foundation Basel, the University Hospital Basel, Abbott, Astra Zeneca, Beckman Coulter, BG medicine, Biomerieux, BRAHMS, Critical Diagnostics, Nanosphere, Roche, Siemens, Singulex, Sphingotec, 8sense as well as speaker/consulting honoraria from Abbott, Alere, Astra Zeneca, Biomerieux, BMS, Boehringer Ingelheim, BRAHMS, Cardiorentis, Eli Lilly, Novartis, Roche, Sanofi, Siemens, and Singulex. Dr Squire has received research grants from Novartis AG and Servier as well as speaker/consulting honoraria from Novartis. The authors declare that they have no conflict of interest.

OpenAccess This article is distributed under the terms of the Creative Commons Attribution 4.0 International License (http://creativeco mmons.org/licenses/by/4.0/), which permits unrestricted use, distribution, and reproduction in any medium, provided you give appropriate credit to the original author(s) and the source, provide a link to the Creative Commons license, and indicate if changes were made.

\section{References}

1. Owan TE, Hodge DO, Herges RM et al (2006) Trends in prevalence and outcome of heart failure with preserved ejection fraction. N Engl J Med 355(3):251-259

2. Meta-analysis Global Group in Chronic Heart F (2012) The survival of patients with heart failure with preserved or reduced left 
ventricular ejection fraction: an individual patient data metaanalysis. Eur Heart J 33(14): 1750-1757

3. Cheng JM, Akkerhuis KM, Battes LC et al (2013) Biomarkers of heart failure with normal ejection fraction: a systematic review. Eur J Heart Fail 15(12):1350-1362

4. Anand IS, Rector TS, Cleland JG et al (2011) Prognostic value of baseline plasma amino-terminal pro-brain natriuretic peptide and its interactions with irbesartan treatment effects in patients with heart failure and preserved ejection fraction: findings from the I-PRESERVE trial. Circ Heart Fail 4(5):569-577

5. Tschöpe C, Birner C, Böhm M et al (2018) Heart failure with preserved ejection fraction: current management and future strategies: expert opinion on the behalf of the nucleus of the "Heart Failure Working Group" of the German Society of Cardiology (DKG). Clin Res Cardiol 107:1-19

6. Arbit B, Marston N, Shah K et al (2016) Prognostic usefulness of proenkephalin in stable ambulatory patients with heart failure. Am J Cardiol 117(8):1310-1314

7. Doehner W, von Haehling S, Suhr J et al (2012) Elevated plasma levels of neuropeptide proenkephalin a predict mortality and functional outcome in ischemic stroke. J Am Coll Cardiol 60(4):346-354

8. Ng LL, Sandhu JK, Narayan H et al (2014) Proenkephalin and prognosis after acute myocardial infarction. J Am Coll Cardiol 63(3):280-289

9. Ng LL, Squire IB, Jones DJ et al (2017) Proenkephalin, renal dysfunction, and prognosis in patients with acute heart failure: a GREAT network study. J Am Coll Cardiol 69(1):56-69

10. Shah KS, Taub P, Patel M et al (2015) Proenkephalin predicts acute kidney injury in cardiac surgery patients. Clin Nephrol 83(1):29-35

11. Siong Chan DC, Cao TH, Ng LL (2018) Proenkephalin in heart failure. Heart Fail Clin 14(1):1-11

12. Denning GM, Ackermann LW, Barna TJ et al (2008) Proenkephalin expression and enkephalin release are widely observed in nonneuronal tissues. Peptides 29(1):83-92

13. Ernst A, Kohrle J, Bergmann A (2006) Proenkephalin A 119-159, a stable proenkephalin A precursor fragment identified in human circulation. Peptides 27(7):1835-1840

14. Lofman I, Szummer K, Dahlstrom U et al (2017) Associations with and prognostic impact of chronic kidney disease in heart failure with preserved, mid-range, and reduced ejection fraction. Eur J Heart Fail 19(12):1606-1614

15. Ponikowski P, Voors AA, Anker SD et al (2016) 2016 ESC guidelines for the diagnosis and treatment of acute and chronic heart failure: the task force for the diagnosis and treatment of acute and chronic heart failure of the European Society of Cardiology (ESC)Developed with the special contribution of the Heart Failure Association (HFA) of the ESC. Eur Heart J 37(27):2129-2200

16. Schiller NB, Shah PM, Crawford M et al (1989) Recommendations for quantitation of the left ventricle by two-dimensional echocardiography. American Society of Echocardiography Committee on Standards, Subcommittee on Quantitation of Two-Dimensional Echocardiograms. J Am Soc Echocardiogr 2(5):358-367

17. Singh A, Greenwood JP, Berry C et al (2017) Comparison of exercise testing and CMR measured myocardial perfusion reserve for predicting outcome in asymptomatic aortic stenosis: the PRognostic Importance of MIcrovascular Dysfunction in Aortic Stenosis (PRIMID AS) Study. Eur Heart J 38(16):1222-1229

18. Gulati A, Ismail TF, Jabbour A et al (2013) Clinical utility and prognostic value of left atrial volume assessment by cardiovascular magnetic resonance in non-ischaemic dilated cardiomyopathy. Eur J Heart Fail 15(6):660-670

19. Ng LL, Geeranavar S, Jennings SC et al (2004) Diagnosis of heart failure using urinary natriuretic peptides. Clin Sci 106(2):129-133

20. Pencina MJ, D’Agostino RB, Steyerberg S EW (2011) Extensions of net reclassification improvement calculations to measure usefulness of new biomarkers. Stat Med 30(1):11-21

21. Jobs A, Schwind J, Katalinic A, Babaev V, Tilz RR, Rausch S, Thiele H, Eitel I, Eitel C (2018) Prognostic significance of atrial fibrillation in acute decompensated heart failure with reduced versus preserved ejection fraction. Clin Res Cardiol. https://doi. org/10.1007/s00392-018-1321-4 (Epub ahead of print, PMID: 29987595)

22. Wienbergen H, Pfister O, Hochadel M, Fach A, Backhaus T, Bruder O, Remppis BA, Maeder MT, von Scheidt W, Pauschinger M, Senges J, Hambrecht R (2018) Long-term effects of iron deficiency in patients with heart failure with or without anemia: the RAID-HF follow-up study. Clin Res Cardiol. https://doi. org/10.1007/s00392-018-1327-y (Epub ahead of print, PMID: 30003365)

23. Pencina MJ, D'Agostino RB, Vasan RS (2010) Statistical methods for assessment of added usefulness of new biomarkers. Clin Chem Lab Med 48:1703-1711

24. Holaday JW (1983) Cardiovascular effects of endogenous opiate systems. Annu Rev Pharmacol Toxicol 23:541-594

25. Nencini P, Paroli E (1981) The lipolytic activity of met-enkephalin, leu-enkephalin, morphine, methadone and naloxone in human adipose tissue. Pharmacol Res Commun 13(6):535-540

26. Brestoff JR, Kim BS, Saenz SA et al (2015) Group 2 innate lymphoid cells promote beiging of white adipose tissue and limit obesity. Nature 519(7542):242-246 